\title{
CÁC YẾU TỐ ẢNH HƯ ỞNG ĐẾN QUYẾT ĐỊNH MUA HÀNG NGẪU HỨNG QUA TRUYỀN HÌNH CỦA KHÁCH HÀNG TẠI CÔNG TY BEST BUY VIETNAM
}

\author{
HÀ NAM KHÁNH GIAO \\ Trường Đại học Tài Chính - Marketing - khanhgiaohn@yahoo.com \\ NGUYẼ̃N TH!̣ HỒNG MƠ \\ Trường Đại học Tài Chính - Marketing - monguyenhong240787@gmail.com \\ (Ngày nhận: 11/01/2017; Ngày nhận lại: 03/09/2017; Ngày duyệt đăng: 25/09/2017)
}

\section{TÓM TẮT}

Nghiên cứu kiểm định mức độ tác động của các yếu tố ảnh hưởng đến quyết định mua hàng ngẫu hứng qua truyền hình của khách hàng tại công ty Best Buy Việt Nam $(\mathrm{BBVN})$, bằng việc khảo sát 560 khách hàng. Phương pháp phân tích Cronbach's Alpha, phân tích EFA, CFA cùng với phân tích cấu trúc tuyến tính SEM được sử dụng với phương tiện SPSS và AMOS.

Kết quả cho thấy mức độ ảnh hưởng của các yếu tố đến Thôi thúc mua hàng ngẫu hứng- theo tầm quan trọng giảm dần: Thuộc tính công ty và sản phẩm, Thời lượng xem chương trình mua sắm qua truyền hình, Sức hút chương trình và khách mời. Thôi thúc mua hàng ngẫu hứng tác động yếu hơn Cảm nhận rủi ro đến Quyết định mua hàng ngẫu hứng qua truyền hình. Nghiên cứu đề ra một số hàm ý quản trị cho các nhà quản lý công ty BBVN nhằm phục vụ khách hàng tốt hơn.

Từ khóa: Công ty Best Buy Việt Nam; Quyết định mua hàng ngẫu hứng qua truyền hình; Thôi thúc mua hàng ngẫu hứng.

\section{Factors affecting consumers' impulsive buying decision on television at Best Buy Vietnam}

\section{ABSTRACT}

This research aims to investigate factors affecting consumers' impulsive buying decision on TV at Best Buy Vietnam (BBVN). The study uses a combination of Cronbach's Alpha, EFA, CFA, and Structural Equation Modelling (SEM) methods to collect data from 560 consumers, then the data were analyzed using SPSS and AMOS.

The result reveals 03 factors affecting Urge to buy impulsively in descending order of importance including: Characteristics of company and products, Time spent watching online shopping programs, and Attraction of program host and celebrity. The impact of Urge to buy impulsively on TV Impulsive Buying Decision are weaker than that of Risk Impression. Accordingly, the research suggests some solutions for BBVN Management to better serve their consumers.

Keywords: Best Buy Vietnam; Impulsive buying decision on TV; Urge to buy impulsively.

\section{Tổng quan}

Hiện nay, bên cạnh các chợ truyền thống, các cửa hàng, siêu thị và trung tâm thương mại, ngày càng xuất hiện nhiều hình thức bán hàng hiện đại như bán hàng qua internet, bán hàng qua truyền hình. Những thay đổi trong hệ thống bán lẻ đã tạo điều kiện cho mua hàng ngẫu hứng nảy sinh và phát triển. Hình thức mua sắm qua truyền hình (MSQTH) phát triển trong những năm gần đây tại Việt Nam, chỉ cần xem tivi, điện thoại tới tổng đài đặt hàng, người tiêu dùng (NTD) mua được sản phẩm (SP) ngay tại nhà, do đó NTD dễ dàng bị kích thích hành vi mua hàng ngẫu hứng.

Hiện đã có rất nhiều công ty gia nhập vào thị trường MSQTH như: Best Buy Việt Nam (BBVN), Home Shopping Việt Nam (HSV), TV Shopping, SCJ Life On, Co.op Home Shopping, Lotte Đất Việt Home Shopping, Happy Shopping,... Tỷ lệ người đã mua hàng 
qua kênh mua sắm trên truyền hình là rất thấp, khoảng $2 \%$ (dddn, 2012). Như là một chương trình mới, hiệu suất và tốc độ tăng trưởng của MSQTH rất đáng chú ý; song MSQTH ở Việt Nam vẫn là con đường dài để trở thành một mô hình mua sắm rộng khắp.

Có mặt tại Việt Nam từ năm 2002, BBVN là doanh nghiệp tiên phong trong lĩnh vực MSQTH. Trong hơn 10 năm qua, công ty luôn thực hiện chiến lược nâng cao chất lượng SP và dịch vụ, mang lại các giải pháp, sự tiện nghi hữu ích phục vụ cho cuộc sống hàng ngày cho NTD. Nhưng trong những năm gần đây, doanh thu của công ty có chiều hướng giảm mạnh, nguyên nhân có thể do sự cạnh tranh gay gắt, và hậu quả của mua hàng ngẫu hứng qua truyền hình tại BBVN đối với NTD. Nghiên cứu này nhằm xác định và đo lường mức độ tác động của các yếu tố ảnh hưởng đến thôi thúc mua hàng ngẫu hứng, và quyết định mua hàng ngẫu hứng qua truyền hình (MHNHQTH) của khách hàng $(\mathrm{KH})$ tại $\mathrm{BBVN}$, từ đó đề xuất các hàm ý quản trị giúp $\mathrm{BBVN}$ điều chỉnh chính sách kinh doanh của mình.

\section{Cơ sở lý luận}

\section{Hành vi mua hàng ngẫu hứng}

Theo Rook \& Hoch (1985), mua hàng ngẫu hứng xảy ra khi NTD trải nghiệm một cảm giác bất chợt, mang tính hối thúc mua một cái gì đó ngay lập tức. Theo Karbasivar \& Yarahmadi (2011), sự ngẫu hứng mua này thể hiện trạng thái tình cảm khá phức tạp và có thể tạo ra những mâu thuẫn nhất định trong suy nghĩ, tình cảm của NTD. Theo Beatty \& Ferrell (1988), mua ngẫu hứng là hành vi mua ngay lập tức không có ý định trước mua một SP cụ thể nào, hành vi xảy ra sau khi trải qua một cảm giác thôi thúc mua và nó có xu hướng là tự phát không cần phải trải qua quá trình đánh giá $\mathrm{SP}$. Rook \& Hoch (1985) cho rằng hành vi mua ngẫu hứng là hành vi liên quan đến một mong muốn bất ngờ, hành động tự phát, và là sự mâu thuẫn về tâm lý. Sharma, Sivakumaran \& Marshall (2010), Kacen \& Lee (2002) cho rằng hành vi mua ngẫu hứng là đặc điểm của mua hàng không có kế hoạch với tốc độ quyết định tương đối nhanh chóng, mong muốn sở hữu loại hàng hóa đó ngay lập tức.

\section{Mua sắm qua truyền hình}

MSQTH thường là hình thức thức phát sóng trực tiếp, chiều dài của chương trình thay đổi tùy theo kênh phát sóng và SP. Trong chương trình MSQTH, SP được giới thiệu bởi người dẫn chương trình và thường là khách mời đặc biệt, những người đã sử dụng SP hoặc chuyên gia. Người xem có nhu cầu mua $S P$, sẽ liên hệ qua số điện thoại hiển thị trên màn hình để gặp tổng đài viên (screendigest, 2004).

Theo Hiệp hội marketing Hoa Kỳ, trong các hình thức tiếp thị trực tiếp thì hình thức quảng cáo có hồi đáp có hai hình thức là hợp đồng dài hạn và hợp đồng ngắn hạn. Hợp đồng dài hạn là hình thức sử dụng một khoảng thời gian dài (khoảng 30 phút) để mô tả SP thật chi tiết và hợp đồng ngắn hạn sử dụng khoảng thời gian quảng cáo ngắn (30 giây hay 1 phút) để hỏi những phản hồi của khách hàng tức khắc bằng cách gọi điện thoại hiện trên màn hình hoặc vào Website. Đây cũng là trong những hình thức bán hàng qua truyền hình.

\section{Các yếu tố ảnh hưởng đến hành vi mua hàng ngẫu hứng}

Theo Churchill \& Peter (1998), hành vi mua hàng ngẫu hứng bị ảnh hưởng bởi các yếu tố bên trong: tâm trạng, nhu cầu, ước muốn, sự vui thích, nhận thức, cảm xúc, sự đánh giá và các yếu tố bên ngoài: quang cảnh của cửa hàng, sự trưng bày bên ngoài cửa hàng, sự trưng bày bên trong cửa hàng, dấu hiệu tiếp thị, khuyến mãi.

Kim (2003), nghiên cứu yếu tố ảnh hưởng đến hành vi mua hàng ngẫu hứng của sinh viên với SP trang phục may mặc, cho thấy có 4 yếu tố ảnh hưởng đến mua hàng ngẫu hứng: Cách trưng bày bên ngoài cửa hàng, cách trưng bày bên trong cửa hàng, dấu hiệu quảng cáo, dấu hiệu khuyến mãi.

Parboteeah (2005) nghiên cứu hành vi mua ngẫu hứng trực tuyến dựa trên mô hình lý thuyết SOR (Kích thích (Stimulus) - Đối tượng (Organism) - Phản ứng (Response)) cho thấy có 5 yếu tố ảnh hưởng đến mua ngẫu hứng trực tuyến của NTD: tính năng tiện ích và tính bảo mật, giao diện, tính hữu dụng, 
tính hưởng thụ, hành vi của NTD (tiếp cận hoặc né tránh) .

Theo Lee (2008), khi nghiên cứu mối quan hệ giữa phương pháp thuyết phục, nhận thức tương tác xã hội, chương trình và người xem trong môi trường MSQTH và xu hướng MSQTH của phụ nữ lớn tuổi cho thấy có 6 yếu tố ảnh hưởng đến mua hàng ngẫu hứng qua truyền hình: chiến lược thuyết phục, tương tác xã hội, nhận thức sự tham gia của xã hội, sự cô đơn, tâm trạng, nhận thức rủi ro.

Cũng nghiên cứu môi trường MSQTH, Tsai, Chen \& Chen (2008) đã nghiên cứu hành vi mua ngẫu hứng tác động đến quyết định MSQTH tại Đài Loan với nhiều SP khác nhau (thời trang, mỹ phẩm, điện tử, ...). cho thấy có 3 nhóm yếu tố ảnh hưởng đến hành vi mua ngẫu hứng qua truyền hình: các yếu tố cá nhân (cảm nhận rủi ro, xu hướng mua ngẫu hứng, quan điểm MSQTH), các yếu tố tình huống (thời gian xem ti vi, thời gian đi ra ngoài mua sắm), các yếu tố kích thích (khả năng chi trả, tiếp thị, sức hút của chương trình và khách mời nổi tiếng).

Theo nghiên cứu của Nguyễn Thị Tuyết Mai và Rose (2006), các nhân tố văn hóa, nhân tố nhân khẩu học và loại SP ảnh hưởng đến hành vi mua hàng ngẫu hứng của người dân Việt Nam ở hai thành phố lớn đó là Hà Nội và Hồ Chí Minh.
Ở phạm vi nghiên cứu nhỏ hơn, Phan Mai Phương Duyên (2012) khảo sát trên 339 NTD tại thành phố Nha Trang, cho thấy có 2 nhóm yếu tố ảnh hưởng đó là: yếu tố bên trong (tâm trạng cảm xúc của người mua) và nhóm yếu tố bên ngoài (Giá và sự giảm giá, trưng bày $\mathrm{SP}$, người đồng hành mua sắm, quảng cáo và khuyến mãi, thương hiệu, mùa lễ hội).

Mô hình nghiên cứu các yếu tố ảnh hưởng đến quyết định mua hàng ngẫu hứng của khách hàng tại Best Buy Việt Nam

Các biến số được cân nhắc sử dụng là những biến số đã được nhiều nhà nghiên cứu kiểm định và sử dụng rộng rãi, và được điều chỉnh thông qua phương pháp chuyên gia và phỏng vấn nhóm. Tám (08) chuyên gia là những người đã và đang giữ chức vụ là Tổng Giám đốc, phó Tổng Giám đốc, Giám đốc, trưởng phòng tại $\mathrm{BBVN}$ được hỏi ý kiến nhằm gợi ý những thành phần ảnh hưởng đến quyết định mua hàng ngẫu hứng qua truyền hình, và kiểm tra, đánh giá cách sử dụng từ ngữ trong từng biến quan sát. Phỏng vấn nhóm được thực hiện với mười (10) khách hàng đã từng MSQTH tại BBVN ít nhất một lần, các ý kiến thảo luận được ghi thành văn bản và thống nhất thông qua biểu quyết đa số. Kết quả là các biến độc lập và hệ thống thang đo được hình thành, mô hình nghiên cứu đề xuất như trong Hình 1.

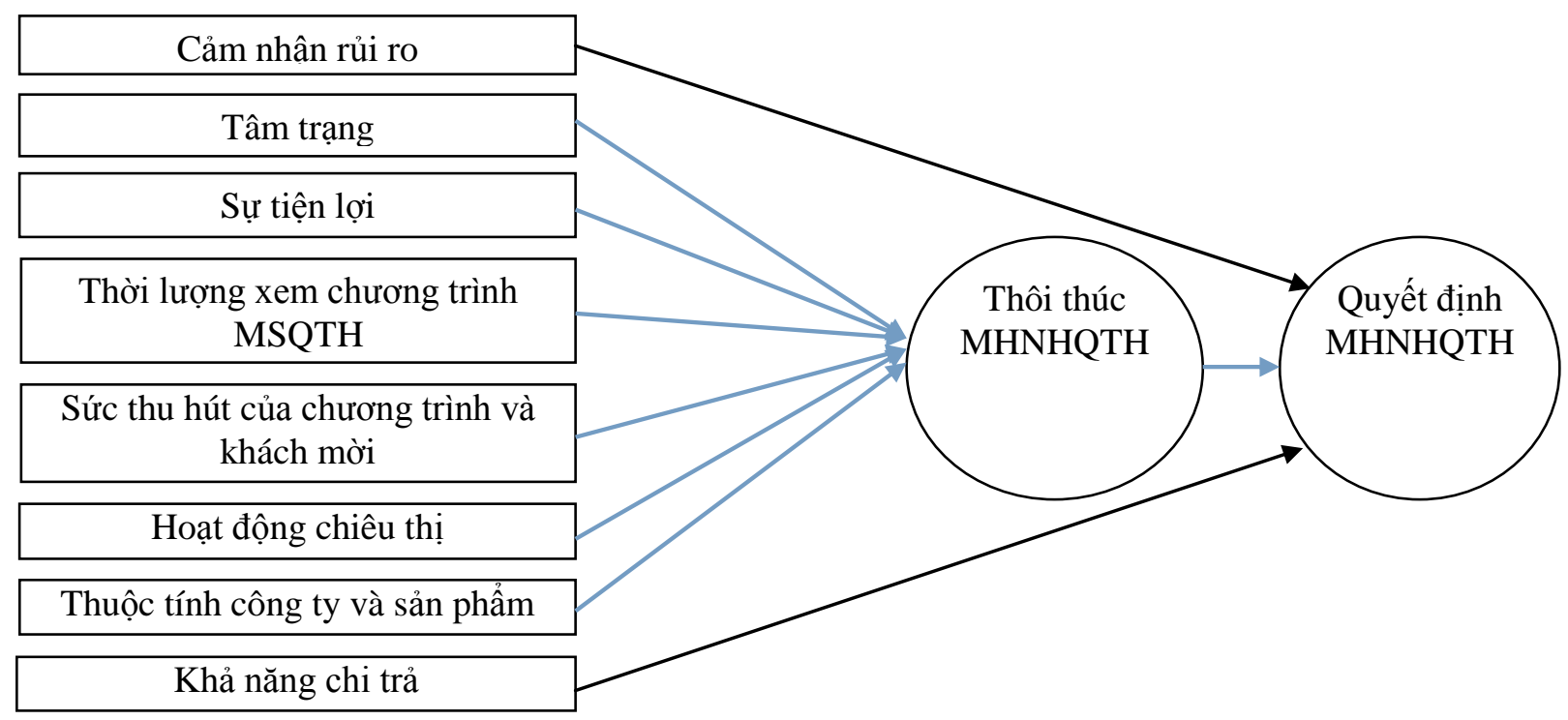

Hình 1. Mô hình những yếu tố ảnh hưởng đến quyết định MHNHQTH tại BBVN Nguồn: Nhóm tác giả đề xuất 
$\mathbf{H}_{1}$ : Cảm nhận rủi ro ảnh hưởng nghịch chiều đến quyết định MHNHQTH của KH tại BBVN

$\mathbf{H}_{2:}$ Tâm trạng ảnh hưởng cùng chiều đến thôi thúc MHNHQTH của KH tại BBVN

$\mathbf{H}_{3}$ : Tính tiện lợi ảnh hưởng cùng chiều đến thôi thúc hành vi MHNHQTH của KH tại BBVN

$\mathbf{H}_{4}$ : Thời lượng xem chương trình MSQTH có ảnh hưởng cùng chiều đến thôi thúc hành vi MHNHQTH của KH tại BBVN

$\mathbf{H}_{5}$ : Sức hút của chương trình và khách mời ảnh hưởng cùng chiều đến thôi thúc hành vi MHNHQTH của KH tại BBVN

H$_{6}$ : Hoạt động chiêu thị ảnh hưởng cùng chiều đến thôi thúc hành vi MHNHQTH của $\mathrm{KH}$ tại BBVN
$\mathbf{H}_{7}$ : Thuộc tính công ty và sản phẩm ảnh hưởng cùng chiều đến thôi thúc MHNHQTH của $\mathrm{KH}$ tại BBVN

H8: Khả năng chi trả ảnh hưởng cùng chiều đến quyết định MHNHQTH của KH tại BBVN

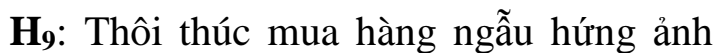
hưởng cùng chiều đến quyết định MHNHQTH của KH tại BBVN

\section{Kết quả nghiên cứu}

Mẫu được lấy theo phương pháp thuận tiện bằng cách phỏng vấn trực tiếp, thư tín và trực tuyến. Tổng số 650 bảng câu hỏi khảo sát được gởi đến những khách hàng từ 18 tuổi trở lên đã từng mua hàng qua truyền hình của BBVN (dựa trên báo cáo bán hàng của công ty), thu về 560 phiếu hợp lệ, đạt $87 \%$.

\section{Bảng 1}

Mô tả thông tin đáp viên

\begin{tabular}{|c|l|c|c|}
\hline & & Số người & Tỷ lệ \\
\hline \multirow{2}{*}{ Giới tính } & Nam & 128 & $22,9 \%$ \\
& Nữ & 432 & $77,1 \%$ \\
\hline \multirow{3}{*}{ Độ tuổi } & Từ 18 - 39 tuổi & 149 & $27 \%$ \\
& Từ 40 - 59 tuổi & 322 & $58 \%$ \\
& Trên 60 tuổi & 89 & $16 \%$ \\
\hline \multirow{3}{*}{ Thu Nhập } & Dưới 5 triệu đồng/tháng & 39 & $7 \%$ \\
& Từ 5 - 10 triệu đồng/tháng & 114 & $20,4 \%$ \\
& Từ 11 - 15 triệu đồng/tháng & 286 & $51,1 \%$ \\
& Trên 15 triệu đồng/tháng & 121 & $21,6 \%$ \\
\hline \multirow{3}{*}{ Khu vực } & Miền Bắc & 197 & $35,2 \%$ \\
& Miền Trung & 113 & $20,4 \%$ \\
& Miền Nam & 250 & $44,6 \%$ \\
\hline
\end{tabular}

Nguồn: Nghiên cúu của nhóm tác giả

Đánh giá độ tin cậy của thang đo bằng Cronbach's Alpha

Kết quả nghiên cứu cho thấy các thang đo đạt chuẩn dao động từ $0,801-0,922$, cần phải loại 3 biến quan sát đó là: $\mathrm{C}_{4}$ thuộc yếu tố Tính tiện lợi, $\mathrm{D}_{1}$ thuộc yếu tố Thời lượng xem chương trình mua sắm và $\mathrm{E}_{4}$ thuộc yếu tố Sức hút của chương trình và khách mời $(\mathrm{E})$ vì có hệ số Cronbach's Alpha lớn hơn hệ số Cronbach's Alpha tổng.

Phân tích EFA những yếu tố tác động đến sự thôi thúc mua hàng ngẫu hứng qua truyền hình tại BBVN

Kết quả phân tích EFA cho ra hệ số KMO 
bằng $0,875(0,5 \leq \mathrm{KMO} \leq 1)$ giải thích được kích thước mẫu phù hợp cho phân tích nhân tố và hệ số Bartlett's có mức ý nghĩa quan sát $0,000<0,05$ (có sự tương quan giữa các biến) đã khẳng định rằng phương pháp phân tích trên là phù hợp, eigenvalue là $1,321(>1)$ và phương sai trích được là $70,811 \%$ (> 50\%) góp phần giải thích được khoảng 70,811\% sự biến thiên của các biến quan sát, như vậy phương sai trích đạt yêu cầu. Các biến quan sát đều có trọng số nhân tố factor loading lớn hơn 0,50 . Kết quả phân tích nhân tố EFA không có biến nào bị loại, có 7 nhóm yếu tố được rút ra (Bảng 2), các biến quan sát đạt yêu cầu của các thang đo này sẽ được đánh giá tiếp theo bằng phân tích yếu tố khẳng định CFA và kiểm định mô hình bằng phân tích SEM.

Phân tích EFA nhũng yếu tố tác động đến quyết định mua hàng ngẫu hứng qua truyền hình tại BBVN
Kết quả phân tích EFA ngay lần đầu đã cho kết quả phù hợp và không loại biến quan sát: $\mathrm{KMO}=0,914(0,5 \leq \mathrm{KMO} \leq 1)$, tổng phương sai trích là $72,243 \%$ góp phần giải thích được khoảng 72,243\%, có 3 nhóm nhân tố được trích tại eigenvalue 1,384 (> 1). Các biến quan sát đều có trọng số nhân tố factor loading lớn hơn 0,50 . Kết quả EFA của các thang đo được trình bày trong Bảng 3 .

Các nhóm yếu tố sau khi phân tích EFA: Cảm nhận rủi ro (A) gồm 5 biến quan sát, Tâm trạng (B) gồm 5 biến quan sát, Tính tiện lợi $(\mathrm{C})$ gồm 3 biến quan sát, Thời lượng xem chương trình MSQTH (D) gồm 3 biến quan sát, Sức hút của chương trình và khách mời (E) gồm 5 biến quan sát, Hoạt động chiêu thị (F) gồm 5 biến quan sát, Thuộc tính của công ty và $S P(G)$ gồm 5 biến quan sát, Khả năng chi trả $(\mathrm{H})$ gồm 4 biến quan sát, Thôi thúc MHNHQTH (I) gồm 4 biến quan sát, Quyết định MHNHQTH (J) gồm 4 biến quan sát.

\section{Bảng 2}

Ma trận các nhân tố trong kết quả xoay EFA

\begin{tabular}{|c|c|c|c|c|c|c|c|c|c|c|}
\hline \multirow{2}{*}{ Biến quan sát } & \multicolumn{10}{|c|}{ Nhóm nhân tố } \\
\hline & 1 & 2 & 3 & 4 & 5 & 6 & 7 & 8 & 9 & 10 \\
\hline $\begin{array}{l}\mathbf{G}_{4} \text { Tôi bị thôi thúc MSQTH của } \\
\text { BBVN vì đây là có danh tiếng }\end{array}$ & 0,855 & & & & & & & & & \\
\hline $\begin{array}{l}\mathbf{G}_{2} \text { Tính năng độc đáo của SP bán } \\
\text { qua truyền hình tại BBVN thôi } \\
\text { thúc tôi suy nghĩ ngay đến việc } \\
\text { mua nó }\end{array}$ & 0,837 & & & & & & & & & \\
\hline
\end{tabular}

$\mathbf{G}_{3}$ Những SP được giới thiệu trên 0.828

kênh MSQTH của BBVN ít thấy

trên thị trường

G $_{5}$ Tôi bị thôi thúc mua hàng của 0,816

BBVN vì đây là có uy tín

$\mathbf{G}_{1}$ SP mới lạ được bán qua truyền 0,798

hình của $B B V N$ thôi thúc tôi

muốn mua nó

$\mathbf{F}_{2}$ Tôi cảm thấy hồi hộp khi thời gian còn lại để đặt hàng với giá tốt nhất hiển thị trên màn hình quảng cáo của $\mathrm{BBVN}$ giảm dần 


\begin{tabular}{|c|c|c|c|c|c|c|c|c|c|c|}
\hline \multirow{2}{*}{ Biến quan sát } & \multicolumn{10}{|c|}{ Nhóm nhân tố } \\
\hline & 1 & 2 & 3 & 4 & 5 & 6 & 7 & 8 & 9 & 10 \\
\hline $\begin{array}{l}\mathbf{F}_{4} \text { Tôi bị cuốn hút bởi thông điệp } \\
\text { quảng cáo "người mua sắm thông } \\
\text { minh" của } \mathrm{BBVN}\end{array}$ & & 0,827 & & & & & & & & \\
\hline
\end{tabular}

$\mathbf{F}_{3}$ Tôi bị cuốn hút bởi thông điệp 0819 quảng cáo "tiết kiệm tiền" của BBVN

$\mathbf{F}_{1}$ Những thông tin đầy đủ về SP 0.802 giới thiệu trong chương trình MSQTH của BBVN thu hút tôi chú ý tới nó

$\mathbf{F}_{5}$ Tôi bị thôi thúc bởi cam kết “được đổi trả miễn phí trong 10 ngày" của BBVN

$\mathbf{E}_{\mathbf{6}}$ Người dẫn chương trình và khách mời trong chương trình 0,962 MSQTH của BBVN tạo cho tôi cảm giác tin tưởng

$\mathbf{E}_{5}$ Người dẫn chương trình và khách mời trong chương trình MSQTH của BBVN tạo cho tôi cảm giác gần gũi

$\mathbf{E}_{3}$ Nhân viên tư vấn của $\mathrm{BBVN}$ rất thân thiện

$\mathbf{E}_{1}$ Những quảng cáo giới thiệu SP trong chương trình MSQTH của $\mathrm{BBVN}$ rất bắt mắt

$\mathbf{E}_{2}$ Nhân viên tư vấn của $\mathrm{BBVN}$ nhiệt tình

$\mathbf{B}_{3}$ Tôi MSQTH của BBVN để 0,800 thay đổi tâm trạng của mình

$\mathbf{B}_{2}$ MSQTH của BBVN là cách để tôi giảm căng thẳng trong cuộc sống

$\mathbf{B}_{1}$ Khi tôi trong trạng thái vui vẻ tôi thích MSQTH của BBVN

$\mathbf{B}_{4}$ Tôi mua SP qua truyền hình của $B B V N$ phụ thuộc vào tâm trạng của tôi lúc đó

$\mathbf{B}_{5}$ Khi trong trạng thái không vui, tôi đi MSQTH của BBVN để làm 0,762 cho mình vui vẻ hơn 


\begin{tabular}{|c|c|c|c|c|c|c|c|c|c|c|}
\hline \multirow{2}{*}{ Biến quan sát } & \multicolumn{10}{|c|}{ Nhóm nhân tố } \\
\hline & 1 & 2 & 3 & 4 & 5 & 6 & 7 & 8 & 9 & 10 \\
\hline $\begin{array}{l}\mathbf{I}_{4} \text { Khi xem các kênh MSQTH của } \\
\text { BBVN, tôi thấy một số thứ muốn } \\
\text { mua mặc dù tôi chưa từng muốn } \\
\text { mua chúng }\end{array}$ & & & & & 0,809 & & & & & \\
\hline $\begin{array}{l}\text { I Khi xem kênh MSQTH của } \\
\text { BBVN, tôi cảm thấy một sự thôi } \\
\text { thúc đột ngột để mua một cái gì } \\
\text { đó }\end{array}$ & & & & & 0,804 & & & & & \\
\hline $\begin{array}{l}\mathbf{I}_{2} \text { Nếu chương trình MSQTH của } \\
\text { BBVN giới thiệu một SP mới mà } \\
\text { tôi quan tâm, tôi sẵn sàng mua } \\
\text { ngay }\end{array}$ & & & & & 0,801 & & & & & \\
\hline
\end{tabular}

$\mathbf{I}_{3}$ Khi xem kênh MSQTH của 0,784 BBVN, tôi cảm thấy có sự thôi thúc bất ngờ để mua những thứ mà tôi chưa từng lên kế hoạch

$\mathbf{C}_{1}$ Mua hàng qua truyền hình tại 0,773 công ty BBVN rất dễ dàng

$\mathrm{C}_{2}$ Mua hàng qua truyền hình tại công ty BBVN rất thuận tiện

$\mathbf{C}_{3}$ Tôi có thể mua sắm tại nhà trên kênh MSQTH của BBVN

$\mathbf{D}_{\mathbf{3}}$ Khi đang xem truyền hình nếu 0,747 thấy chương trình MSQTH của $\mathrm{BBVN}$ tôi thường dừng lại xem

$\mathbf{D}_{2}$ Tôi thường xem chương trình MSQTH của BBVN

$\mathbf{D}_{4}$ Tôi thường coi hết chương trình quảng cáo MSQTH của BBVN

$\mathrm{A}_{3}$ Tôi cho rằng $\mathrm{SP}$ mua qua 0,846 truyền hình của $\mathrm{BBVN}$ sẽ không phù hợp với tôi

$\mathrm{A}_{4}$ Tôi cho rằng $\mathrm{SP}$ mua qua truyền hình của $\mathrm{BBVN}$ khó trả lại sau khi mua

$\mathrm{A}_{1}$ Tôi cho rằng $\mathrm{SP}$ mua qua truyền hình của BBVN không giống như hiển thị trên quảng cáo

$\mathrm{A}_{5}$ Tôi cho rằng $\mathrm{MSQTH}$ tại 0,712

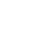




\begin{tabular}{|c|c|c|c|c|c|c|c|c|c|c|}
\hline \multirow{2}{*}{ Biến quan sát } & \multicolumn{10}{|c|}{ Nhóm nhân tố } \\
\hline & 1 & 2 & 3 & 4 & 5 & 6 & 7 & 8 & 9 & 10 \\
\hline $\begin{array}{l}\mathbf{J}_{4} \text { Khi có nhu cầu về hàng hóa, tôi } \\
\text { sẽ nghĩ đến hình thức MSQTH } \\
\text { của } B B V N\end{array}$ & & & & & & & & & 0,802 & \\
\hline $\begin{array}{l}\mathbf{J}_{2} \text { Tôi sẽ tiếp tục MSQTH của } \\
\text { BBVN trong thời gian tới }\end{array}$ & & & & & & & & & 0,781 & \\
\hline $\begin{array}{l}\mathbf{J}_{1} \text { Tôi sẽ MSQTH của BBVN } \\
\text { ngay khi tôi có ý định mua mà } \\
\text { không cần phải cân nhắc }\end{array}$ & & & & & & & & & 0,780 & \\
\hline $\begin{array}{l}\mathbf{J}_{\mathbf{3}} \text { Tôi rất sẵn lòng giới thiệu cho } \\
\text { mọi người để lựa chọn các SP } \\
\text { được bán qua truyền hình của } \\
\text { BBVN }\end{array}$ & & & & & & & & & 0,742 & \\
\hline
\end{tabular}

$\mathbf{H}_{2}$ Khi có tiền mặt, tôi sẵn sàng mua hàng truyền hình tại $\mathrm{BBVN}$

$\mathbf{H}_{3}$ Khi tôi có một khoản tiết kiệm dành cho mua sắm, tôi sã̃n sàng chọn MSQTH tại BBVN

$\mathbf{H}_{1}$ Có thu nhập cao tôi sã̃n sàng MSQTH tại BBVN

$\mathbf{H}_{4}$ Tôi sẵ sàng dùng thẻ ghi nợ để MSQTH tại BBVN

\section{KMO}

Giá trị Eigen

Tổng phương sai trích

Nguồn: Nghiên cứu của nhóm tác giả

\section{Kết quả phân tích nhân tố khẳng định CFA}

Đánh giá lại các thang đo bằng hệ số tin cậy tổng hợp và phân tích nhân tố khẳng định CFA dựa vào dữ liệu của nghiên cứu chính thức với kích thước mẫu $\mathrm{n}=560$. Kết quả CFA từng phần cho thấy $\mathrm{GFI} \geq 0,9, \mathrm{TLI} \geq 0,9$, $\mathrm{CFI} \geq 0,9, \mathrm{CMIN} / \mathrm{df} \leq 3$, RMSEA $\leq 0,08$ đều đạt yêu cầu (Nguyễn Đình Thọ \& Nguyễn Thị Mai Trang, 2011).
Kết quả CFA toàn phần, sau khi liên kết $\mathrm{E}_{2}$ và $\mathrm{E}_{3}$ để hiệu chỉnh hiệp phương sai, cho thấy mô hình có 814 bậc tự do, Chi-bình phương là $1333,904(\mathrm{p}=0,000)$; GFI $=0,901 ; \mathrm{TLI}=$ 0,961 ; CFI $=0,965$ (GFI, TLI, CFI > 0,9); Chibình phương/df $=1,639$; RMSEA $=0,034$ (CMIN/df < 3, RMSEA < 0,08), các chỉ số đều đạt yêu cầu. Như vậy, mô hình hoàn toàn phù hợp với dữ liệu thị trường (Nguyễn Đình Thọ \& Nguyễn Thị Mai Trang, 2011) (Hình 2). 


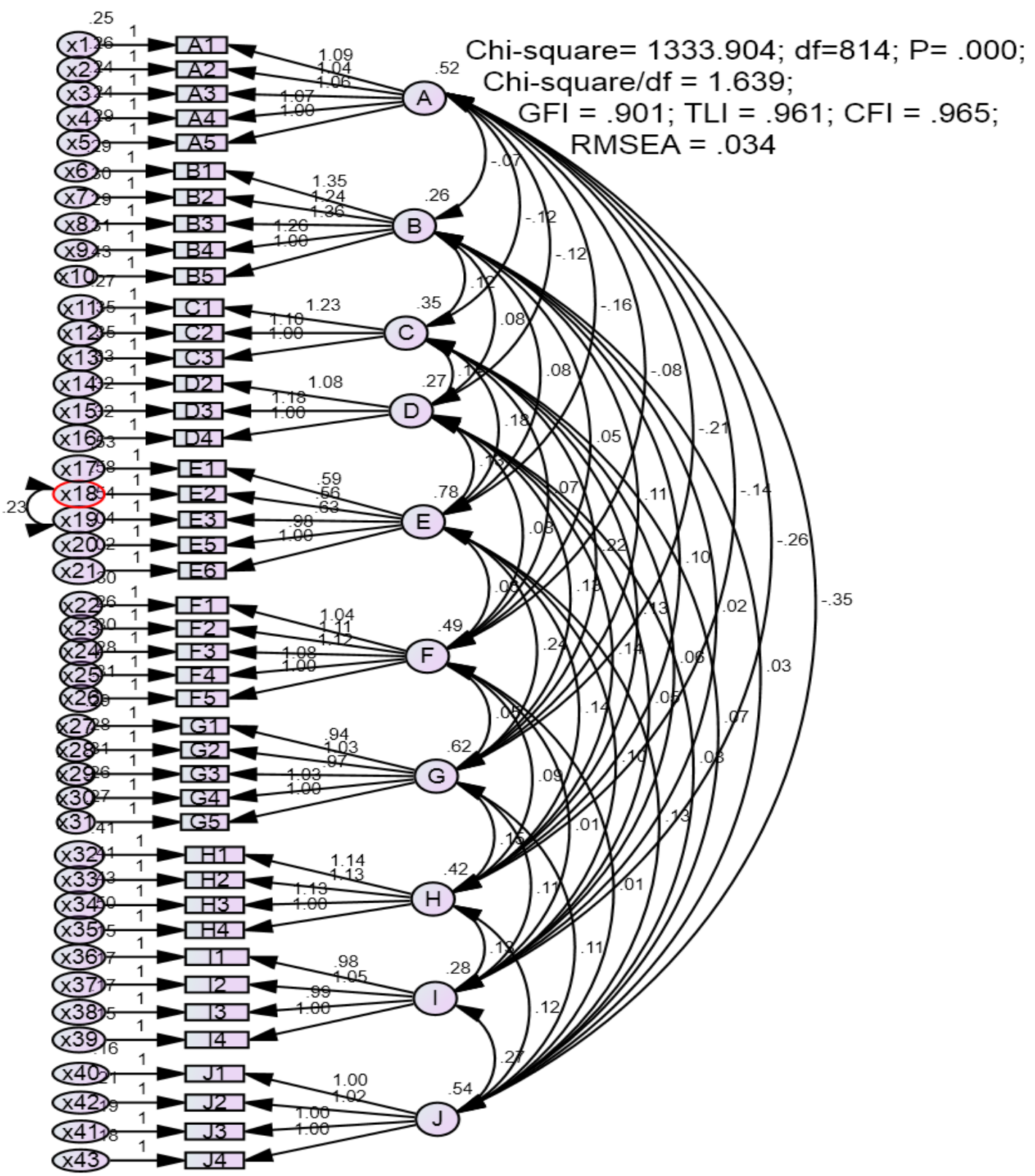

Hình 2. Mô hình CFA tới hạn

Nguồn: Nghiên cứu của nhóm tác giả

\section{Bảng 3}

Bảng tóm tắt kết quả kiểm định thang đo

\begin{tabular}{|c|c|c|c|c|c|c|}
\hline $\begin{array}{l}\text { Khái } \\
\text { niệm }\end{array}$ & $\begin{array}{l}\text { Số biến } \\
\text { quan sát }\end{array}$ & $\begin{array}{c}\text { Độ tin cậy } \\
\text { Cronbach's } \\
\text { Alpha }\end{array}$ & $\begin{array}{c}\text { Độ tin cậy } \\
\text { tổng hợp } \rho C\end{array}$ & $\begin{array}{l}\text { Tổng phương } \\
\text { sai trích } \rho V C\end{array}$ & $\begin{array}{l}\text { Trung } \\
\text { bình } \lambda\end{array}$ & $\begin{array}{l}\text { Giá trị hội tụ } \\
\text { và phân biệt }\end{array}$ \\
\hline A & 5 & 0,918 & 0,918 & $69,217 \%$ & 0,832 & \multirow{4}{*}{ thỏa mãn } \\
\hline B & 5 & 0,859 & 0,861 & $55,448 \%$ & 0,742 & \\
\hline $\mathrm{C}$ & 3 & 0,801 & 0,801 & $57,396 \%$ & 0,756 & \\
\hline $\mathrm{D}$ & 3 & 0,774 & 0,747 & $49,577 \%$ & 0,704 & \\
\hline
\end{tabular}




\begin{tabular}{|c|c|c|c|c|c|c|}
\hline $\begin{array}{c}\text { Khái } \\
\text { niệm }\end{array}$ & $\begin{array}{c}\text { Số biến } \\
\text { quan sát }\end{array}$ & $\begin{array}{c}\text { Độ tin cậy } \\
\text { Cronbach's } \\
\text { Alpha }\end{array}$ & $\begin{array}{c}\text { Độ tin cậy } \\
\text { tổng họ̣p } \boldsymbol{\rho} \boldsymbol{C}\end{array}$ & $\begin{array}{c}\text { Tổng phương } \\
\text { sai trích } \boldsymbol{\rho} \boldsymbol{V} \boldsymbol{C}\end{array}$ & $\begin{array}{c}\text { Trung } \\
\text { bình } \boldsymbol{\lambda}\end{array}$ & $\begin{array}{c}\text { Giá trị hội tụ } \\
\text { và phân biệt }\end{array}$ \\
\hline $\mathrm{E}$ & 5 & 0,884 & 0,868 & $58,476 \%$ & 0,738 & \\
\hline $\mathrm{F}$ & 5 & 0,906 & 0,906 & $65,838 \%$ & 0,811 \\
\hline $\mathrm{G}$ & 5 & 0,916 & 0,916 & $68,583 \%$ & 0,828 \\
\hline $\mathrm{H}$ & 4 & 0,820 & 0,822 & $53,614 \%$ & 0,732 \\
\hline $\mathrm{I}$ & 4 & 0,876 & 0,877 & $63,967 \%$ & 0,800 \\
\hline $\mathrm{J}$ & 4 & 0,922 & 0,922 & $74,613 \%$ & 0,864 & \\
\hline
\end{tabular}

Nguồn: Nghiên cứu của nhóm tác giả

Kiểm định giá trị phân biệt: Bảng 4 cho thấy các hệ số tương quan của từng cặp khái niệm kèm với sai lệch chuẩn của các thang đo đều khác với 1 ở độ tin cậy $95 \%$, đạt mức ý nghĩa thống kê (tất cả $\mathrm{p}$ đều bằng 0,000 ). Do đó, các biến quan sát dùng để đo lường các khái niệm nghiên cứu đều đạt được giá trị phân biệt.

\section{Bảng 4}

Hệ số tương quan giữa các khái niệm

\begin{tabular}{|c|c|c|c|c|c|c|}
\hline \multicolumn{3}{|c|}{$\mathbf{r}$} & Estimate & $\operatorname{SE}=\operatorname{SQRT}\left(\left(1-\mathbf{r}^{2}\right) /(n-2)\right)$ & $\mathrm{CR}=(1-\mathrm{r}) / \mathrm{SE}$ & P-value \\
\hline B & $\langle-->$ & $\mathrm{C}$ & 0,400 & 0,039 & 15,464 & 0,000 \\
\hline B & $\langle-->$ & $\mathrm{D}$ & 0,284 & 0,041 & 17,640 & 0,000 \\
\hline $\mathrm{B}$ & $<-->$ & $\mathrm{E}$ & 0,183 & 0,042 & 19,631 & 0,000 \\
\hline $\mathrm{B}$ & $<->$ & $\mathrm{F}$ & 0,136 & 0,042 & 20,601 & 0,000 \\
\hline B & $\langle-->$ & $\mathrm{G}$ & 0,266 & 0,041 & 17,987 & 0,000 \\
\hline B & $\langle->$ & $\mathrm{I}$ & 0,070 & 0,042 & 22,023 & 0,000 \\
\hline $\mathrm{C}$ & $\langle-->$ & $\mathrm{D}$ & 0,448 & 0,038 & 14,585 & 0,000 \\
\hline $\mathrm{C}$ & $<-->$ & $\mathrm{E}$ & 0,345 & 0,040 & 16,485 & 0,000 \\
\hline $\mathrm{C}$ & $<-->$ & $\mathrm{F}$ & 0,176 & 0,042 & 19,773 & 0,000 \\
\hline $\mathrm{C}$ & $<-->$ & $\mathrm{G}$ & 0,464 & 0,038 & 14,293 & 0,000 \\
\hline $\mathrm{C}$ & $<-->$ & $\mathrm{I}$ & 0,185 & 0,042 & 19,590 & 0,000 \\
\hline D & $<-->$ & $\mathrm{E}$ & 0,282 & 0,041 & 17,678 & 0,000 \\
\hline D & $\langle-->$ & $\mathrm{F}$ & 0,213 & 0,041 & 19,027 & 0,000 \\
\hline D & $<-->$ & $\mathrm{G}$ & 0,320 & 0,040 & 16,954 & 0,000 \\
\hline D & $<-->$ & I & 0,187 & 0,042 & 19,550 & 0,000 \\
\hline $\mathrm{E}$ & $<-->$ & $\mathrm{F}$ & 0,089 & 0,042 & 21,605 & 0,000 \\
\hline $\mathrm{E}$ & $\langle->$ & G & 0,338 & 0,040 & 16,616 & 0,000 \\
\hline
\end{tabular}


Hà Nam Khánh Giao và cộng sự. Tạp chí Khoa học Đại học Mở Thành phố Hồ Chí Minh, 57(6), 87-102 97

\begin{tabular}{|c|c|c|c|c|c|c|}
\hline \multicolumn{3}{|c|}{$\mathbf{r}$} & Estimate & $\mathrm{SE}=\operatorname{SQRT}\left(\left(1-\mathrm{r}^{2}\right) /(\mathrm{n}-2)\right)$ & $\mathrm{CR}=(1-\mathrm{r}) / \mathrm{SE}$ & P-value \\
\hline $\mathrm{E}$ & $<-->$ & I & 0,221 & 0,041 & 18,868 & 0,000 \\
\hline $\mathrm{F}$ & $\langle-->$ & $\mathrm{G}$ & 0,095 & 0,042 & 21,475 & 0,000 \\
\hline $\mathrm{F}$ & $<-->$ & I & 0,034 & 0,042 & 22,832 & 0,000 \\
\hline G & $<-->$ & I & $\underline{0,264}$ & 0,041 & 18,025 & 0,000 \\
\hline $\mathrm{B}$ & $\langle-->$ & A & $-0,199$ & 0,041 & 28,901 & 0,000 \\
\hline $\mathrm{C}$ & $\langle-->$ & A & $-0,288$ & 0,041 & 31,771 & 0,000 \\
\hline $\mathrm{D}$ & $\langle-->$ & A & $-0,310$ & 0,040 & 32,548 & 0,000 \\
\hline $\mathrm{E}$ & $\langle->$ & A & $-0,247$ & 0,041 & 30,399 & 0,000 \\
\hline $\mathrm{F}$ & $\langle-->$ & A & $-0,160$ & 0,042 & 27,759 & 0,000 \\
\hline G & $\langle-->$ & A & $-0,371$ & 0,039 & 34,875 & 0,000 \\
\hline $\mathrm{H}$ & $\langle->$ & A & $-0,310$ & 0,040 & 32,548 & 0,000 \\
\hline I & $<-->$ & A & $-0,676$ & 0,031 & 53,726 & 0,000 \\
\hline $\mathrm{J}$ & $\langle-->$ & A & $-0,660$ & 0,032 & 52,195 & 0,000 \\
\hline B & $<-->$ & $\mathrm{H}$ & 0,296 & 0,040 & 17,410 & 0,000 \\
\hline $\mathrm{C}$ & $\langle-->$ & $\mathrm{H}$ & 0,326 & 0,040 & 16,841 & 0,000 \\
\hline $\mathrm{D}$ & $<-->$ & $\mathrm{H}$ & 0,402 & 0,039 & 15,427 & 0,000 \\
\hline $\mathrm{E}$ & $<-->$ & $\mathrm{H}$ & 0,252 & 0,041 & 18,259 & 0,000 \\
\hline $\mathrm{F}$ & $\langle-->$ & $\mathrm{H}$ & 0,200 & 0,041 & 19,287 & 0,000 \\
\hline G & $<-->$ & $\mathrm{H}$ & 0,286 & 0,041 & 17,601 & 0,000 \\
\hline B & $<-->$ & $\mathrm{J}$ & 0,072 & 0,042 & 21,978 & 0,000 \\
\hline $\mathrm{C}$ & $<-->$ & $\mathrm{J}$ & 0,161 & 0,042 & 20,081 & 0,000 \\
\hline $\mathrm{E}$ & $<-->$ & $\mathrm{J}$ & 0,195 & 0,042 & 19,388 & 0,000 \\
\hline $\mathrm{F}$ & $<-->$ & $\mathrm{J}$ & 0,029 & 0,042 & 22,947 & 0,000 \\
\hline G & $\langle-->$ & $\mathrm{J}$ & 0,196 & 0,042 & 19,368 & 0,000 \\
\hline $\mathrm{H}$ & $\langle-->$ & $\mathrm{J}$ & 0,262 & 0,041 & 18,064 & 0,000 \\
\hline I & $<-->$ & $\mathrm{J}$ & 0,694 & 0,030 & 10,040 & 0,000 \\
\hline $\mathrm{D}$ & $<-->$ & $\mathrm{J}$ & 0,199 & 0,041 & 19,307 & 0,000 \\
\hline I & $<->$ & $\mathrm{H}$ & 0,382 & 0,039 & 15,796 & 0,000 \\
\hline x19 & $<->$ & $\mathrm{x} 18$ & 0,416 & 0,038 & 15,170 & 0,000 \\
\hline
\end{tabular}

Ghi chú: r: hệ số tuoong quan; CR: giá trị tới hạn

SE: độ lệch chuẩn; P-Value: múc ý nghĩa

Nguồn: Nghiên cứu của nhóm tác giả 
Kiểm định giá trị hội tụ: Kết quả nghiên cứu cho thấy các trọng số chuẩn hóa đều lớn hơn 0,5 và có ý nghĩa thống kê $(\mathrm{P}<0,05)$. Do đó, chúng đạt được giá trị hội tụ (Nguyễn Đình Thọ \& Nguyễn Thị Mai Trang, 2011).

Kết quả cho thấy thang đo Sức hút chương trình và khách mời $(\mathrm{E})$ không đạt được tính đơn hướng, nhưng các yêu cầu về giá trị phương sai trích, giá trị hội tụ, giá trị phân biệt đều đạt. Các thang đo còn lại đều đạt đầy đủ các yêu cầu về độ tin cậy và giá trị: tính đơn hướng, giá trị hội tụ, giá trị phân biệt và phương sai trích.

Kiểm định độ thích hợp của mô hình nghiên cứu và các giả thuyết
Kết quả phân tích cấu trúc tuyến tính cho thấy mô hình có 822 bậc tự do với giá trị thống kê Chi-square là 1582,769 (p=0,000), GFI $=0,887 ;$ TLI $=0,944 ;$ CFI $=0,949$; RMSEA =0,041; chỉ tiêu GFI để đánh giá mức độ phù hợp với dữ liệu thị trường chưa thỏa, do đó cần xem xét mối tương quan giữa sai số của biến quan sát để cải thiện các giá trị trên. Ta cần xem xét ý nghĩa thống kê của các mối quan hệ ở độ tin cậy $90 \%$. Dựa vào bảng kết quả nghiên cứu (Bảng 5), cần loại các yếu tố Tính tiện lợi $(\mathrm{C})$, Hoạt động chiêu thị $(\mathrm{F})$, Khả năng chi trả $(\mathrm{H})$, và Tâm trạng $(\mathrm{B})$ do có $\mathrm{p}>0,1$ ra khỏi mô hình vì không có ý nghĩa thống kê.

\section{Bảng 5}

Kết quả kiểm định mối quan hệ nhân quả giữa các khái niệm trong mô hình nghiên cứu

\begin{tabular}{|lcl|c|c|c|c|}
\hline & & & Estimate & S.E. & C.R. & P \\
\hline I & $<---~$ & B & $\mathbf{- 0 , 0 4 7}$ & $\mathbf{0 , 0 5 5}$ & $\mathbf{- 0 , 8 6 5}$ & $\mathbf{0 , 3 8 7}$ \\
\hline I & $<---~$ & C & $\mathbf{0 , 0 0 2}$ & $\mathbf{0 , 0 5 9}$ & $\mathbf{0 , 0 3 0}$ & $\mathbf{0 , 9 7 6}$ \\
\hline I & $<---$ & D & 0,173 & 0,062 & 2,785 & 0,005 \\
\hline I & $<---$ & E & 0,072 & 0,029 & 2,463 & 0,014 \\
\hline I & $<---~$ & F & $\mathbf{- 0 , 0 1 9}$ & $\mathbf{0 , 0 3 6}$ & $\mathbf{- 0 , 5 2 6}$ & $\mathbf{0 , 5 9 9}$ \\
\hline I & $<---$ & G & 0,129 & 0,037 & 3,493 & $* * *$ \\
\hline J & $<---$ & A & $-0,395$ & 0,040 & $-9,962$ & $* * *$ \\
\hline J & $<---$ & H & $\mathbf{- 0 , 0 2 6}$ & $\mathbf{0 , 0 4 4}$ & $\mathbf{- 0 , 5 9 6}$ & $\mathbf{0 , 5 5 1}$ \\
\hline J & $<---$ & I & 0,629 & 0,054 & 11,592 & $* * *$ \\
\hline
\end{tabular}

Ghi chú: Estimate: giá trị ước luợng; S.E: Độ lệch chuẩn;

C.R: giá trị tới hạn; P: Mức ý nghĩa

Nguồn: Nghiên cứu của nhóm tác giả

Sau khi loại bỏ các yếu tố $\mathrm{C}, \mathrm{F}, \mathrm{H}, \mathrm{B}$ ra khỏi mô hình nghiên cứu, mô hình có sự cải thiện về thông số Chi-square/df $=2,225$; GFI $=0,922 ;$ TLI $=0,960 ;$ CFI $=0,965 ;$ RMSEA = 0,047 , các chỉ tiêu đánh giá mức độ đều phù hợp với dữ liệu thị trường, có ý nghĩa thống kê vì p-value < 0,1 ở độ tin cậy $90 \%$. Cả bốn yếu tố Thời lượng xem chương trình MSQTH,
Sức hút chương trình và khách mời, Thuộc tính công ty và SP đều ảnh hưởng thuận chiều đến Thôi thúc mua ngẫu hứng, Thôi thúc mua ngẫu hứng ảnh hưởng thuận chiều đến quyết định mua ngẫu hứng, Cảm nhận rủi ro ảnh hưởng ngược chiều đến Quyết định mua hàng ngẫu hứng và các yếu tố đều có ý nghĩa thống kê (Bảng 6). 


\section{Bảng 6}

Kết quả kiểm định mối quan hệ nhân quả giữa các khái niệm (chưa chuẩn hóa)

\begin{tabular}{|lcl|c|c|c|c|}
\hline & & Estimate & S.E. & C.R. & P \\
\hline $\mathbf{I}$ & $<--$ & D & 0,141 & 0,060 & 2,351 & 0,019 \\
\hline $\mathbf{I}$ & $<--$ & E & 0,073 & 0,029 & 2,530 & 0,011 \\
\hline $\mathbf{I}$ & $<--$ & G & 0,128 & 0,034 & 3,739 & $* * *$ \\
\hline $\mathbf{J}$ & $<---$ & A & $-0,392$ & 0,038 & $-10,373$ & $* * *$ \\
\hline $\mathbf{J}$ & $<---$ & I & 0,621 & 0,054 & 11,557 & $* * *$ \\
\hline
\end{tabular}

Nguồn: Nghiên cúu của nhóm tác giả

\section{Bảng 7}

Kết quả kiểm định mối quan hệ nhân quả giữa các khái niệm (chuẩn hóa)

\begin{tabular}{|ccc|c|c|c|c|}
\hline & $\mathbf{r}$ & & Estimate & SE=SQRT((1-r2)/(n-2)) & CR=(1-r)/SE & P-value \\
\hline I & $<---~$ & D & 0,130 & 0,042 & 20,727 & 0,000 \\
\hline I & $<---~$ & E & 0,121 & 0,042 & 20,917 & 0,000 \\
\hline I & $<---$ & G & 0,190 & 0,042 & 19,489 & 0,000 \\
\hline J & $<---$ & A & $-0,417$ & 0,038 & 36,827 & 0,000 \\
\hline J & $<---$ & I & 0,487 & 0,037 & 13,875 & 0,000 \\
\hline
\end{tabular}

Nguồn: Nghiên cứu của nhóm tác giả

Dựa vào Bảng 7 , kết quả chuẩn hóa của mô hình cấu trúc tuyến tính SEM cho thấy đối với yếu tố độc lập: Cảm nhận rủi ro là yếu tố tác động mạnh nhất $(\beta=-0,417)$; tiếp theo là Thuộc tính công ty và SP $(0,190)$; thứ ba là Thời lượng xem chương trình $\operatorname{MSQTH}(0,130)$ và cuối cùng là Sức hút chương trình và khách mời $(0,121)$. Thôi thúc mua hàng ngẫu hứng có $\beta$ là 0,487 .

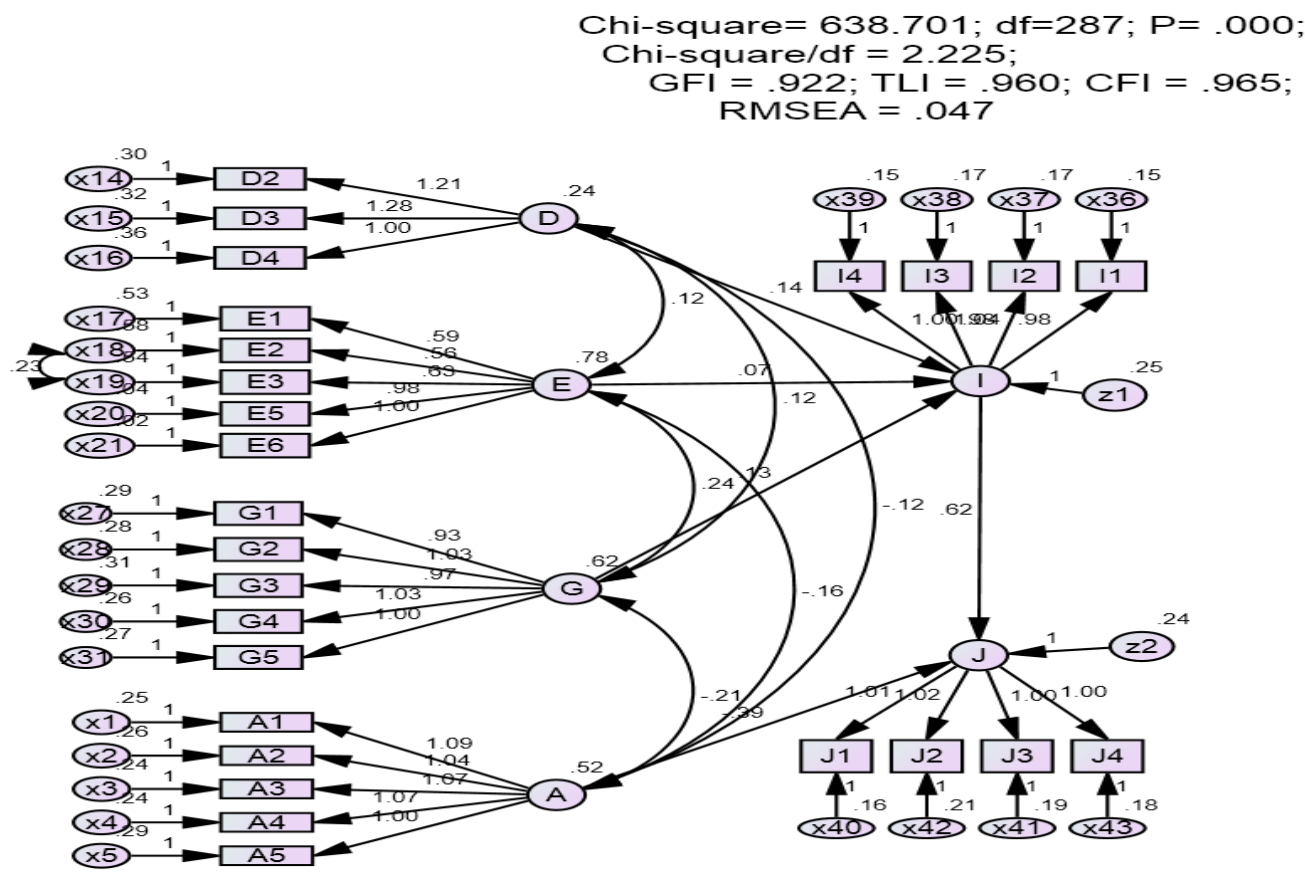

Nguồn: Nghiên cứu của nhóm tác giả

Hình 3. Mô hình cấu trúc tuyến tính SEM 
Ngoài ra, Bảng 8 cho thấy các khái niệm Thời lượng xem chương trình mua sắm, Sức hút chương trình và khách mời $(\mathrm{E})$, Thuộc tính công ty và $\mathrm{SP}(\mathrm{G})$ giải thích được $10,7 \%$ biến thiên của Thôi thúc mua hàng ngẫu hứng, Thôi thúc mua hàng ngẫu hứng và Cảm nhận rủi ro giải thích được $47,6 \%$ biến thiên của Quyết định mua hàng ngẫu hứng qua truyền hình tại BBVN.

\section{Bảng 8}

Hệ số bình phương tương quan bội

\begin{tabular}{|l|c|}
\hline \multicolumn{1}{|c|}{ Nhân tố } & \multicolumn{2}{|c|}{ Estimate } \\
\hline Hành vi mua hàng ngẫu hứng $(\mathrm{I})$ & 0,107 \\
\hline Quyết định mua hàng ngẫu hứng $(\mathrm{J})$ & 0,470 \\
\hline
\end{tabular}

Nguồn: Nghiên cúu của nhóm tác giả

Kết quả kiểm định cho thấy các giả thuyết không được chấp nhận bao gồm $\mathrm{H}_{2}, \mathrm{H}_{3}, \mathrm{H}_{6}, \mathrm{H}_{8}$ vì không có ý nghĩa thống kê, các giả thuyết $\mathrm{H}_{4}$, $\mathrm{H}_{5}, \mathrm{H}_{7}, \mathrm{H}_{1}, \mathrm{H}_{9}$ được chấp nhận. Kết quả kiểm định thang đo cho thấy các thang đo đều đạt độ tin cậy, mô hình phù hợp với dữ liệu thị trường và các giá trị đạt độ tin cậy p-value < 0,1 , do vậy các yếu tố ảnh hưởng đến quyết định mua hàng ngẫu hứng qua truyền hình của $\mathrm{KH}$ tại BBVN tuân theo mô hình thể hiện ở Hình 4.

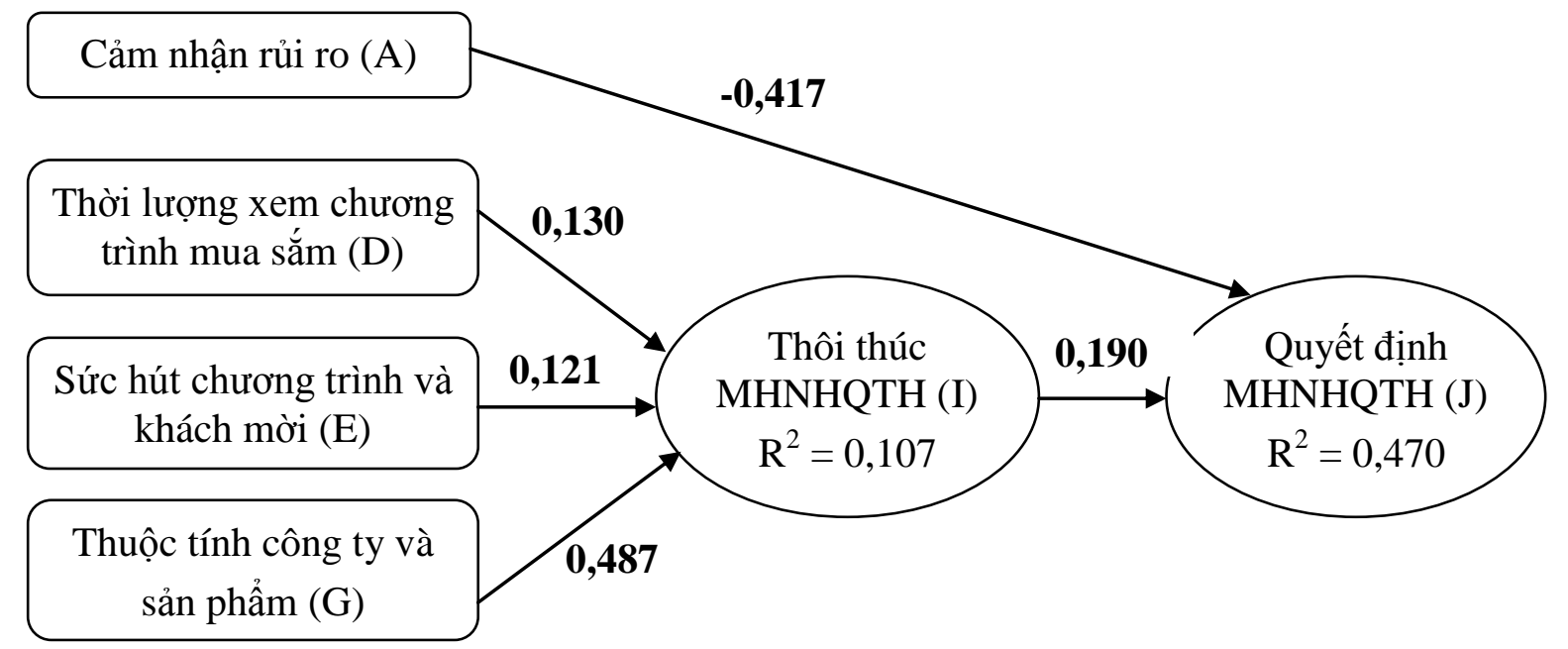

Hình 4. Mô hình các yếu tố ảnh hưởng đến quyết định mua hàng ngẫu hứng qua truyền hình tại Nguồn: Nghiên cúu của nhóm tác giả BBVN hiệu chỉnh

Các yếu tố Tính tiện lợi, Hoạt động chiêu thị, Khả năng chi trả, và Tâm trạng bị loại khỏi mô hình, và cũng là điểm khác biệt của nghiên cứu này, có thể được hiểu như sau: (1) BBVN không có một kênh phát sóng riêng, $\mathrm{KH}$ cần theo dõi lịch phát sóng quảng cáo $\mathrm{SP}$ của BBVN trên các kênh truyền hình, một chương trình quảng cáo $\mathrm{SP}$ của $\mathrm{BBVN}$ dài từ 5 đến 30 phút, $\mathrm{KH}$ phải mất ít nhất 5 phút xem giới thiệu SP và đặt hàng, và mất ít nhất 4 giờ để nhận hàng từ công ty. Trong khi đó, NTD dễ dàng tìm thấy các loại $\mathrm{SP}$ đa dạng từ nhiều nguồn khác nhau, có thể đăng nhập, hoặc mua hàng ở bất cứ đâu một cách thuận tiện, (2) Khả năng chi trả không phải là vấn đề quan tâm lớn khi KH MSQTH tại BBVN do những SP của BBVN hầu hết mang tính chất sử dụng lâu dài (dòng máy tập thể dục, đồ dùng nhà bếp,...); 
và giá bán không quá cao, khoảng 0,5 đến 6 triệu đồng, (3) Với yếu tố tâm trạng và hoạt động chiêu thị: Nguyễn Thị Tuyết Mai \& Rose (2006) đã chỉ ra rằng nhiều người Việt Nam cảm thấy không hài lòng với những lần mua ngẫu hứng. Họ cảm thấy ân hận khi quyết định mua SP không cần thiết hay không phù hợp. Do vậy, họ sẽ cố gắng "thông minh" và "tỉnh táo" hơn trong việc kiểm soát những ngẫu hứng, đặc biệt sẽ cảnh giác hơn với những tác động của người bán hàng.

\section{Kết luận và hàm ý quản trị}

\subsection{Kết luận}

Nghiên cứu này đã kế thừa các lý thuyết nền tảng và kết quả của các nghiên cứu trước đây để xây dựng mô hình các yếu tố ảnh hưởng đến quyết định MHNHQTH của KH tại BBVN, gồm 10 yếu tố với 48 biến quan sát.

Sau khi tiến hành phân tích hệ số tin cậy Cronbach's Alpha, phân tích nhân tố khám phá EFA, phân tích nhân tố khẳng định $\mathrm{CFA}$, mô hình vẫn còn 10 yếu tố, 43 biến quan sát. Kết quả phân tích mô hình cấu trúc tuyến tính SEM lần thứ nhất, các yếu tố Tính tiện lợi, Hoạt động chiêu thị, Khả năng chi trả và Tâm trạng bị loại do không có ý nghĩa thống kê. Kết quả phân tích SEM lần hai cho thấy các yếu tố tác động cùng chiều đến Thôi thúc MHNHQTH, theo thứ tự độ mạnh giảm dần: Thuộc tính công ty và $\mathrm{SP}$, Thời lượng xem chương trình MSQTH, Sức hút chương trình và khách mời. Thôi thúc MHNHQTH tác động yếu đến Quyết định MHNHQTH hơn Cảm nhận rủi ro (tác động ngược chiều). Từ đây, ban quản lý BBVN có thể vận dụng vào kinh doanh hiệu quả nhất. Một số hàm ý quản trị cũng được đề xuất.

\section{2. Đề xuất một số hàm ý quản trị}

Thuộc tính công ty và sản phẩm

BBVN cần không ngừng xây dựng, giữ vững cũng như phát huy danh tiếng cho BBVN, trong đó, việc quan tâm tới chất lượng sản phẩm và dịch vụ là rất quan trọng. BBVN cần luôn cung cấp các sản phẩm mới lạ, có tính năng độc đáo, ít thấy trên thị trường, không nên duy trì dòng sản phẩm quá lâu.
BBVN cần thực hiện đúng các cam kết với khách hàng về hàng hóa và dịch vụ. Ngoài ra, bên cạnh việc thành lập bộ phận sáng tạo nghiên cứu, phát hiện ra nhu cầu tiềm ẩn của NTD cho ra dòng sản phẩm mới và lạ, độc đáo, công ty cần khuyến khích nhân viên đưa ra những ý kiến sáng tạo nhằm thay đổi tính năng hoặc có ý kiến hay về dòng sản phẩm sẽ được giới thiệu bán qua truyền hình.

\section{Thời lượng xem chương trình mua sắm qua truyền hình}

BBVN cần mở một kênh mua sắm riêng với tần số phát sóng cao (24 giờ/1 ngày), đồng thời hướng tới đối tượng là người cao tuổi, người làm nội trợ, những người dân vùng nông thôn (đặc điểm của những người này là có nhiều thời gian, thường xuyên xem tivi). Ngoài ra, BBVN cần tạo mọi khả năng để khách hàng xem hết chương trình quảng cáo của mình bằng việc phát sóng quảng cáo vào những thời điểm khách hàng nhàn rỗi.

\section{Sức hút chương trình và khách mời}

BBVN cần tạo những quảng cáo hấp dẫn, cuốn hút và gây ấn tượng với khách hàng bằng những hình ảnh và kỹ xảo. $\mathrm{BBVN}$ cần tuyển dụng tư vấn viên qua điện thoại vui vẻ, hòa nhã, giọng nói hay, thuyết phục. BBVN cũng cần thường xuyên mở khóa huấn luyện về giao tiếp trong bán hàng qua điện thoại cho nhân viên tư vấn, ghi âm cuộc gọi để theo dõi chất lượng tư vấn. Việc duy trì khách mời là những chuyên gia, những người nổi tiếng, được nhiều người biết đến và yêu thích, và phù hợp với sản phẩm cũng rất cần thiết đối với BBVN.

\section{Cảm nhận rủi ro}

Trung thực trong bán hàng là cách để tạo niềm tin nơi khách hàng, vậy nên BBVN cần quảng cáo đúng sự thật, không phóng đại công dụng, tính năng của $\mathrm{SP}$. BBVN cần đào tạo đội ngũ tư vấn cũng như theo dõi chất lượng tư vấn đảm bảo tính trung thực khi tư vấn cho khách hàng. BBVN nên thường xuyên huấn luyện và kiểm tra đội ngũ tư vấn về $\mathrm{SP}$, hiểu rõ tính năng, công dụng, nguồn gốc của SP để có thể tự tin tư vấn đúng SP 
khách hàng cần. BBVN cần đưa ra chính sách hoàn trả rõ ràng ngay trong quảng cáo giới thiệu và khi tư vấn bán hàng, hay khi giao hàng cũng cần nhắc lại khách hàng về thông tin đổi trả; thực hiện đúng cam kết với khách hàng về việc hoàn trả; quy trình đổi trả cần đơn giản và nhanh chóng.

\subsection{Hạn chế và hướng nghiên cứu tiếp theo}

Nghiên cứu không tránh khỏi những hạn chế nhất định: (1) Nghiên cứu chủ yếu dựa vào lý thuyết và kế thừa các nghiên cứu nước ngoài, khi áp dụng tại thị trường Việt Nam, cần có những điều chỉnh thang đo phù hợp hơn nữa, (2) Nghiên cứu chỉ được thực hiện tại BBVN, tính khái quát hóa chưa cao, (3) Nghiên cứu chưa thể khai thác hết tất cả các yếu tố tác động đến hành vi MHNHQTH. Đó cũng chính là những gợi ý cho các nghiên cứu tiếp theo

\section{Tài liệu tham khảo}

Beatty, S.E., \& Ferrell, M.E. (1998). Impulse buying: Modeling its precursors. Journal of Retailing, 74(2), 169-191.

Churchill, G.A., \& Peter, J.P. (1998). Marketing: Creating value for customers. Boston: Irwin/McGraw-Hill.

Diễn đàn doanh nghiệp điện tử (2012). Thi truoòng. Retrieved from Diển đàn doanh nghiệp website http://dddn.com.vn/thi-truong on May 20, 2015.

Kacen, J. J., \& Lee. J. A. (2002). The influence of culture on consumers impulsive buying. Journal of consumer psychology, 12(2), 163-176.

Karbasivar, A., \& Yarahmadi, H. (2011). Evaluating Effective Factors on Consumer Impulse Buying Behavior. Asian Journal of Business Management Studies, 2(4), 174-181.

Kim, J. (2003). College student's apparel impulse behaviors in relation to visual merchandising. Master of Science, American Intercontinental University, Georgia.

Lee, MS. (2008). Television shopping: the effect of persuasive strategies on parasocial interaction, subjective wellbeing, and impulse buying tendency among older women. Pro Quest Information and Learning Compan, Iowa State University.

Nguyễn Đình Thọ \& Nguyễn Thị Mai Trang (2011). Nghiên cứu khoa học marketing - Úng dụng mô hình cấu trúc tuyến tính SEM. Nhà xuất bản Lao động - Xã hội, TPHCM.

Nguyen, T.T.M., \& Jerman, R. (2006). An exploratory Investigation into outcomers of impulse Buying in VietNam, a Transitional Economy. Asia Pacific Advance in Consumer Research, Provo, UT: Association for Consumer Research.

Parboteeah, V. (2005). A model of online impulse buying: An empirical. Washington State University.

Phan Mai Phương Duyên (2012). Các nhân tố ảnh hưởng đến hành vi mua hàng ngẫu hứng tại Thành phố Nha Trang. Thư viện Đại học Nha Trang.

Rook, D.W., \& Hoch, S.J. (1985). Consuming impulse. Advances in Consumer Research, 12, 23-27.

Screen, D. L. (2004). TV shopping and T-commerce in Europe. Goldmedia GmbH, 8(1), 17.

Sharma, P., Sivakumaran, B., \& Marshall, R. (2010). Impulse buying and variety seeking: A trait-correlates perspective. Journal of Business Research, 63(3), 276-283.

Tsai, D., Wei-Wei, C., \& Hsien-Kai, C. (2008). Factors Influencing Irrational Buying: The Case of Television Shopping. Cyberpsychology and Behavior, 1-19. 